\title{
Gridding and Discretization For Divergence Form (Semiconductor-Like) PDEs
}

\author{
MING Y. KAO, DONALD J. ROSE and HAI SHAO* \\ Dept. Computer Science, Duke University, Durham, NC 27708-0129
}

\begin{abstract}
We develop the box method for solving partial differential equations in the divergence (or conservation law) form. We first use graphic theoretical approaches to generate grids, i.e. partition the governing domain into boxes. Then we apply Green's theorem box-wisely and the constant $-j$ assumption edge-pair-wisely to obtain the discretization system of equations, which lead to the numerical solution to the problem. We show that the box method is inherently more efficient than the traditional finite element method for linear (or convection-diffusion) problems in 1 and 2 dimensions. We also present some potential thoughts on implementing the box method for problems in higher dimensions and with nonlinearity.
\end{abstract}

Keywords: Box method, Grid generation, Voronoi diagram, Divergence form partial differential equations, Boundary value problems

\section{INTRODUCTION}

In this paper we will discuss gridding and discretization for a PDE (or PDE system) of the form.

$$
-\nabla \cdot j+f=0
$$

where

$$
j=\left(j_{1}, j_{2}\right)^{T},
$$

and $j$, the current density function, and $f$, the source density function, specified as.

$$
\begin{gathered}
j_{k}=j_{k}\left(x, y, u_{x}, u_{y}, u\right), \quad k=1,2, \\
f=f\left(x, y, u_{x}, u_{y}, u\right),
\end{gathered}
$$

can take various forms. Our intention will be to consider increasingly more general cases of $j$. Here we focus on the cases where

$$
\begin{array}{r}
j=\alpha \nabla u+\beta u, \alpha=\left(\begin{array}{rr}
\alpha_{11}(x, y) & \alpha_{12}(x, y) \\
\alpha_{21}(x, y) & \alpha_{22}(x, y)
\end{array}\right), \\
\beta=\left(\begin{array}{l}
\beta_{1}(x, y) \\
\beta_{2}(x, y)
\end{array}\right) .
\end{array}
$$

Note that (1.4) arises in the continuity equations of the drift-diffusion semiconductor equations; here $\beta=\mu E, \mu$ a mobility and $E$ the electric field.

We are mainly interested in the cases where $\mathrm{j}$ has a dominant convection term and the region on which the PDE is defined has complex geometry. Rather than the traditional finite element methods, we use two graphs for partitioning (gridding) the region, namely, the Voronoi diagram and the Delaunay triangulation, which are straight line duals from the geometric point of view.

Our approach to discretization (in 2D) will be to use the "box method" (finite volume and finite box

\footnotetext{
* Corresponding author.
} 
methods $[1,5])$ using Voronoi boxes for appropriate grid points in the region specifying the PDE. By using the divergence theorem, this transforms (1.1) to the operational equation

$$
-\oint_{\partial B_{i}} j \cdot n d s+\int_{B_{i}} f=0,
$$

where $B_{i}$ is a box containing grid point $p_{i}$ with boundary $\partial B_{i}$. After approximating the integrals in (1.5) on each box $B_{i}$ associated with the unknown $u_{i}$, we obtain the coupled system of nonlinear (or linear) algebraic equations representing the discretization. We have found that discretization and gridding are tightly coupled to each other in a way that discretization imposes a set of specifications onto gridding while limitations of gridding schemes may require adjustments on discretization strategies. It is important to understand how the trade-offs are made among the generality of the problem, the numerical quality of the discretization, and the complexity of grid construction.

\section{DISCRETIZATION}

The transient extension of equation (1.1),

$$
\frac{d u}{d t}-\nabla \cdot j+f=0
$$

where $j$ and $f$ are defined as in (1.2) and (1.3), is easily handled. Hence most of our exposition will deal with the PDE in the form of (1.1)-(1.3).

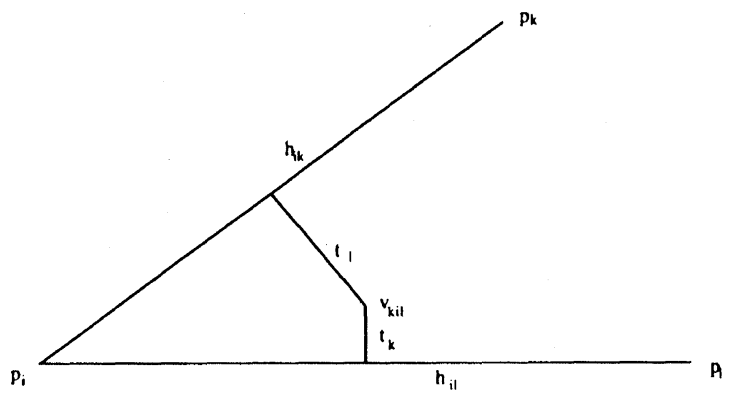

Case 1
In order to discretize (1.5), we need to approximate the integrals. We will derive a discretization based on the constant $j$ assumption, namely, that $j$ is constant pairwise on the edges of the Delaunay triangles dual to the sides of box $B_{i}$. Suppose that $B_{i}, B_{l}$, and $B_{k}$ are neighbors pairwisely, and therefore $\bar{p}_{\bar{i}} \bar{p}_{\bar{i}}$ and $\bar{p}_{\bar{i}} \bar{p}_{\bar{k}}$ form an edge pair. Depending on whether the circumcenter of $\Delta p_{i} p_{l} p_{k}$ lies inside or outside the triangle, we need to approximate the integrals in (1.5) in different ways (Figure 1).

In particular, there may be more errors in case 2 of Figure 1 because we approximate integrals outside the edge pair by using the interior information. Let $J_{[k i l]}$ and $F_{[k i l]}$ denote approximations of the part of the line integral of $j$ and the area integral of $f$ in (1.5) that is contained in the edge pair $\left(p_{i} p_{l} p_{i} p_{k}\right)$. In the case where $\alpha$ and $\beta$ are constant, we have the following result :

$$
\begin{aligned}
& J_{[k i l]]}=\left\|t_{l}\right\| n_{i k}^{T} j \pm\left\|t_{k}\right\| n_{i l}^{T} j \\
& ("+" \text { for case } 1 \text { and " }-" \text { for case 2) } \\
& =\frac{1}{2 \mid \operatorname{det}(H)}\left(\begin{array}{c}
h_{k l}^{T} h_{i l} \\
h_{l k}^{T} h_{i k}
\end{array}\right)^{T} \\
& H \alpha H^{-1}\left(\begin{array}{cc}
B\left(-\lambda_{i j}\right) u_{j} & -B\left(\lambda_{i j}\right) u_{i} \\
B\left(-\lambda_{i k}\right) u_{k} & -B\left(\lambda_{i k}\right) u_{i}
\end{array}\right),
\end{aligned}
$$

$$
\begin{aligned}
& F_{[k i l]}=\frac{f}{4}\left(\left\|t_{l}\right\|\left\|h_{i k}\right\| \pm\left\|t_{k}\right\|\left\|h_{i l}\right\|\right) \\
& ("+" \text { for case } 1 \text { and " }-" \text { for case 2) } \\
& =\frac{f\left(\left\|h_{i k}\right\|^{2} h_{k l}^{T} h_{i l}+\left\|h_{i l}\right\|^{2} h_{l k}^{T} h_{i k}\right)}{4 \operatorname{det}(H)}
\end{aligned}
$$

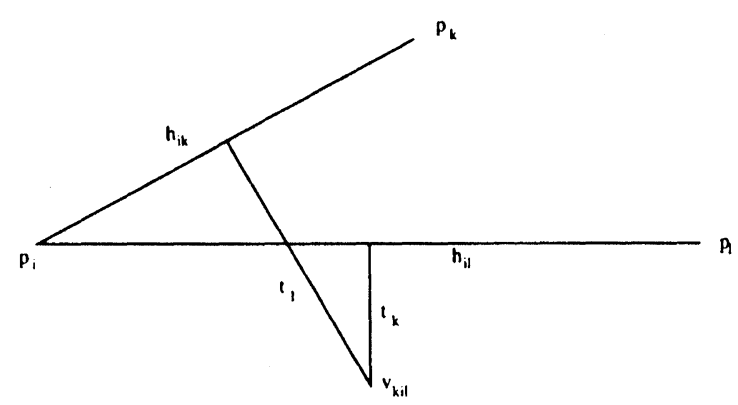

Case 2

FIGURE 1 Examples of edge pairs 
where $h_{i l}=\left(\begin{array}{c}x_{l}-x_{i} \\ y_{l}-y_{i}\end{array}\right), h_{i k}=\left(\begin{array}{c}x_{k}-x_{i} \\ y_{k}-y_{i}\end{array}\right)$,

$$
H=\left(h_{i l} h_{i k}\right)^{T}, n=\frac{h}{\|h\|}, \lambda=h^{T} \alpha^{-1} \beta, \text { and }
$$

$B(\lambda)=\lambda /\left(e^{\lambda}-1\right)$. If $\alpha$ and $\beta$ vary smoothly over space, we can evaluate them at the circumcenter of $\Delta p_{k} p_{i} p_{l}$ (or the edge midpoints), and the above form can still be applied to achieve first order accuracy. This allows us to assemble for each box the parts of the line integral and the area integral which, in turn, relate the variable $u_{i}$ to the other $u_{k}$ corresponding to Delaunay neighbors, say $p_{k}$, of the box mesh point $p_{i}$. We see that the constant $j$ discretization is a natural generalization of the Scharfetter-Gummel $[1,5,6]$ discretization used in semiconductor modeling.

When $j$ is linear, as in (1.4), the constant $j$ discretization produces a linear system to solve with interesting matrix properties which are closely related to those of the gridding output. For instance, a lower bound on the angles of the Delaunay triangulation gives a lower bound on the conditional number of the matrix, and an upper bound on the angles is crucial to the accuracy of approximation in (2.2). These considerations motivate the following gridding scheme.

\section{GRIDDING}

Suppose the region on which the PDE in (1.1) is specified is a connected open set $\Omega$ of $\mathscr{R}^{2}$. We partition $\Omega$ via the Voronoi scheme based on a set of grid points $P=\left\{p_{1}, \ldots, p_{n}\right\}$. For each grid point $p_{i}$, we find its Voronoi box $B_{i} \subseteq \Omega$ where each point is closer to $p_{i}$ than to any other grid points in $P$. The collection of the Voronoi boxes, $v=\left\{B_{1} \ldots, B_{n}\right\}$, is called the Voronoi diagram of $P$. In addition, the straight-line dual planar graph of the Voronoi diagram yields the Delaunay triangulation $D$ of $P$, with the grid points in $P$ being the vertices of the triangles. The edges of the Delaunay triangles are perpendicularly bisected by the sides of the Voronoi boxes. The union of the Voronoi diagram and the Delaunay triangulation yields a grid on $\Omega$

Most of the previous results on grid generation have been oriented to the finite element method. Two schemes that have been widely studied are quadtree based techniques [2] and Delaunay based techniques $[3,4]$. Our grid however has to meet a more specific set of conditions that are required by our discretization scheme.

1. The angles of all the triangles of $D$ must be bounded both below and above by constants $\theta_{\text {min }}$ and $\theta_{\max }$ respectively. The constant $\theta_{\min }$ must be large enough to ensure that a well conditioned algebraic system can be obtained from the discretization; $\theta \max$ should be small (ideally $<90^{\circ}$ ) so that the effect of case 2 in Figure 1 is negligible.

2. The edges of all the triangles of $D$ must be bounded above by $h_{0}$, so that the discretization can reach certain required accuracy.

3. No Voronoi boxes in $\nu$ cross $\partial \Omega$.

We call a grid that satisfies these conditions a quasiuniform grid. Based on some of the previous work [2, $3,4]$, we have implemented our own scheme for generating quasiuniform grids on a region with general boundary geometries. Here is a brief description of our algorithm:

1. Decompose the given region $\Omega$ into subregions by using the quad-tree based techniques [2].

2. Generate points in each subregion according to its relative position to the boundary of $\Omega$.

3. Apply the filtering process of Miller et al [3] so that the Delaunay triangulation of the remaining points meets condition 1 above.

4. Generate the Voronoi diagram and the Delaunay triangulation for the remaining points.

Let $P$ be the set of output grid points of this algorithm. We can show that the size of $P,|P|$, is optimal, i.e.,

$$
|P| \leq c \frac{S(\Omega)}{h_{0}^{2}},
$$

for some constant c $>0$, where $S(\Omega)$ denotes the area of $\Omega$. Furthermore, the points of $P$ are uniformly distributed on $\Omega$ with density approximately $h_{0}^{-2}$. In the cases where $\alpha$ and $\beta$, or the source density function, $f$, are ill behaved at some points, we can locally refine the grid so that these points will be included in $P$ and conditions 1-3 above for the grid will still hold. 


\section{EXAMPLES}

Here we show some of our results on solving two simple cases of (1.1) with $j=\alpha \nabla u+\beta u$. The first case (see Figure 2) is an extended 1D problem where $\Omega$ is specified as a square region with vertices located at $( \pm 1, \pm 1)$. Here $\alpha$ is a $2 \times 2$ identity matrix, $\beta=\left(\begin{array}{c}10 \\ 0\end{array}\right)$, and $f \equiv 0$. The boundary conditions are all Dirichlet and fit the function $\phi(x, y)$ which can be solved exactly from

$$
\begin{gathered}
\frac{\partial}{\partial x}\left(\frac{\partial \phi}{\partial x}+\beta_{1} \phi\right)=0, \quad \frac{\partial \phi}{\partial y}=0, \\
\phi(-1, y) \equiv 1, \phi(1, y) \equiv 0 .
\end{gathered}
$$

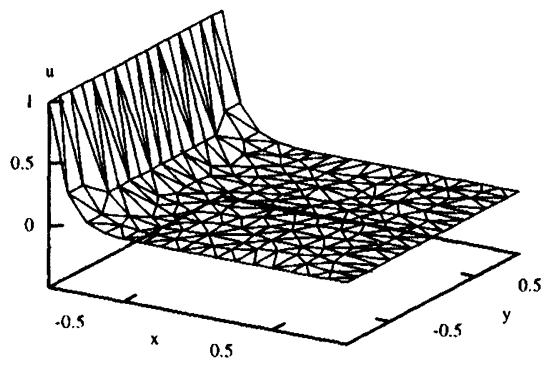

FIGURE $21 \mathrm{D}$ dominant convection case

In the second case (see Figure 3), we solve the Poisson equation $\nabla^{2} u-1$ on the disk $\left\{(x, y) \mid x^{2}+y^{2} \leq 1\right\}$. The boundary conditions are Dirichlet and identically zero.

We see that our results fit well to the analytical solutions for both cases. For the dominant convection case, our box method gives a much better approximation to the analytical solution than the finite difference or the finite element methods.

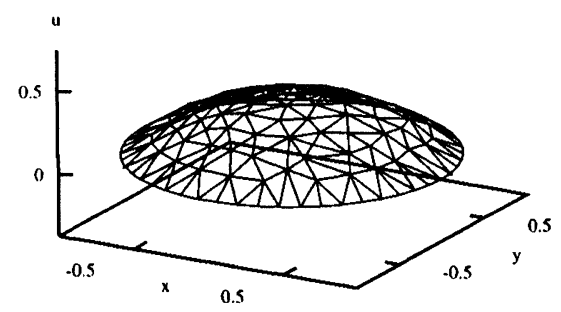

FIGURE 3 Polar symmetric case

\section{CONCLUSION}

We have shown that the constant $j$ discretization is efficient and convenient for solving the 2D boundary value problems with $j$ linear in terms of $\nabla u$ and $u$. We are extending our study to the cases where $j$ is nonlinear and generalizing our 2D schemes to 3D.

\section{References}

[1] R. E. Bank, D. J. Rose, and W. Fichtner, "Numerical Methods for Semiconductor Simulation," SIAM J. Scientific Stat Comp. 4,416-435, 1983.

[2] M. Bern, D. Eppstein, and J. R. Gilbert, "Provably Good Mesh Generation," FOCS90, 231-241, IEEE, 1990.

[3] G. L. Miller, D. Talmor, S. Teng, and N. Walkington, "A Delaunay Based Numerical Method for Three Dimensions : Generation, Formulation, and Partition," FOCS95, 683-692, IEEE, 1995.

[4] J. Ruppert, "A New and Simple Algorithm for Quality 2-Dimensional Mesh Generation," Third Annual ACM-SIAM Symposium Theory of Computing, 404-413, ACM, 1986.

[5] S. Selberherr, Analysis and Simulation of Semiconductor Devices, Springer-Verlag, Wien, New York, 1984.

[6] D. Scharfetter and H. Gummel, "Large-signal analysis of a silicon Read diode oscillator", IEEE Solid-State Electron, 16, 64-77, 1973.

\section{Biographies}

Ming-Yang Kao received his B.S. in mathematics from National Taiwan University and his Ph.D. in computer science from Yale University. He is currently on the computer science faculty at Duke University. His research interests include computational finance, parallel computing and online algorithms among others.

Donald J. Rose was born in Santa Ana, CA, on May 25, 1944. He received the B.A. degree in mathematics from the University of California at Berkeley in 1966, and the M.A. and Ph.D. degrees in applied mathematics from Harvard University, Cambridge, MA, in 1967 and 1970, respectively. From 1970 to 1984, Dr. Rose held research positions at the University of Denver, Harvard University, Vanderbilt University, and AT\&T Bell Laboratories. In 1984, he joined the Computer Science Department at Duke University, Durham, NC, where he investigates problems involving numerical analysis and scientific computation. Dr. Rose is a member of SIAM, AMS, MAA, ACM and IEEE. 
Hai Shao received his B.S. degree in physics and mathmatics from Grodon College, Wenham, MA, in 1994, after which he joined the Ph.D. program of the
Computer Science Department at Duke University, Durham, NC. He is currently a research assistant for Dr. Rose. 

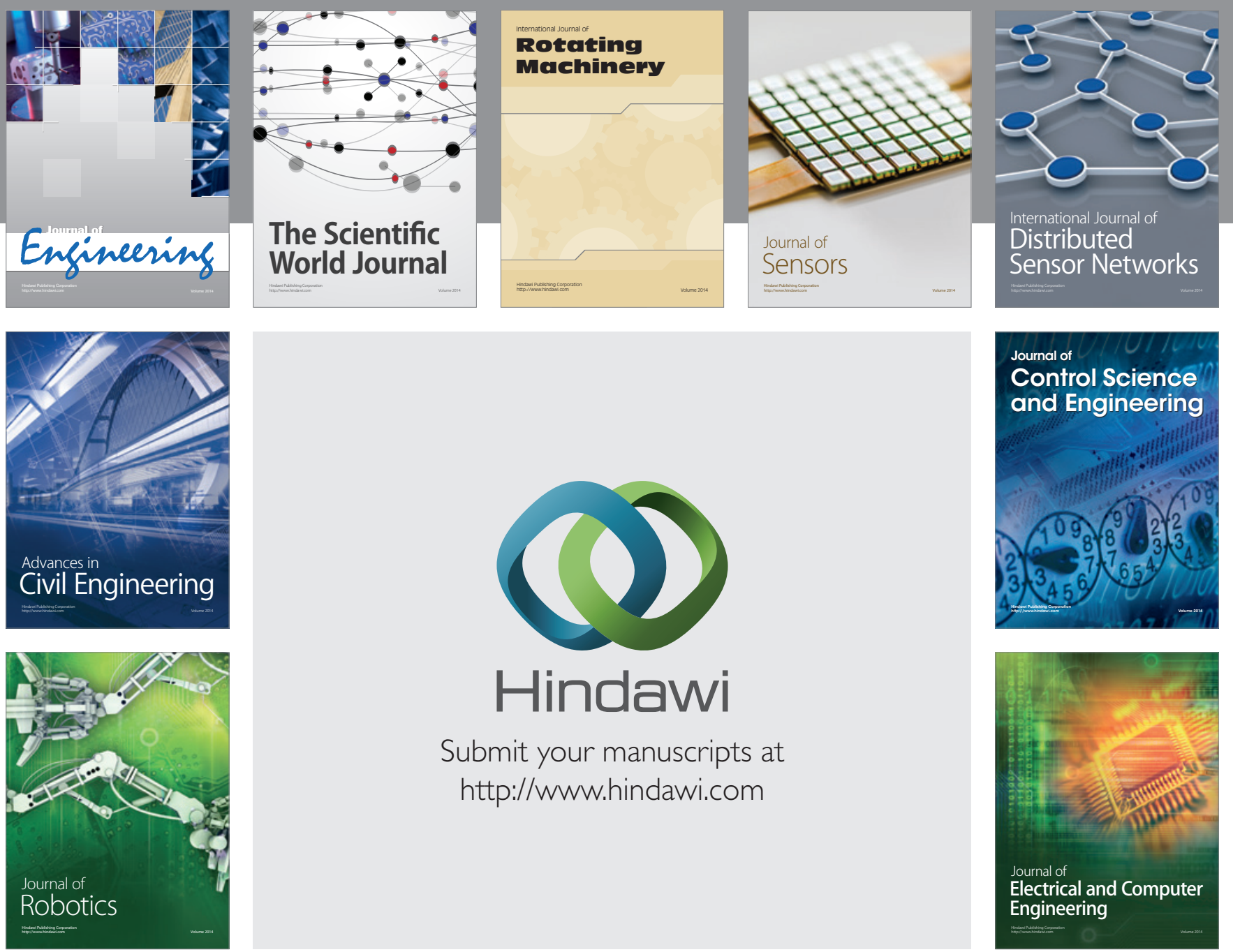

Submit your manuscripts at

http://www.hindawi.com
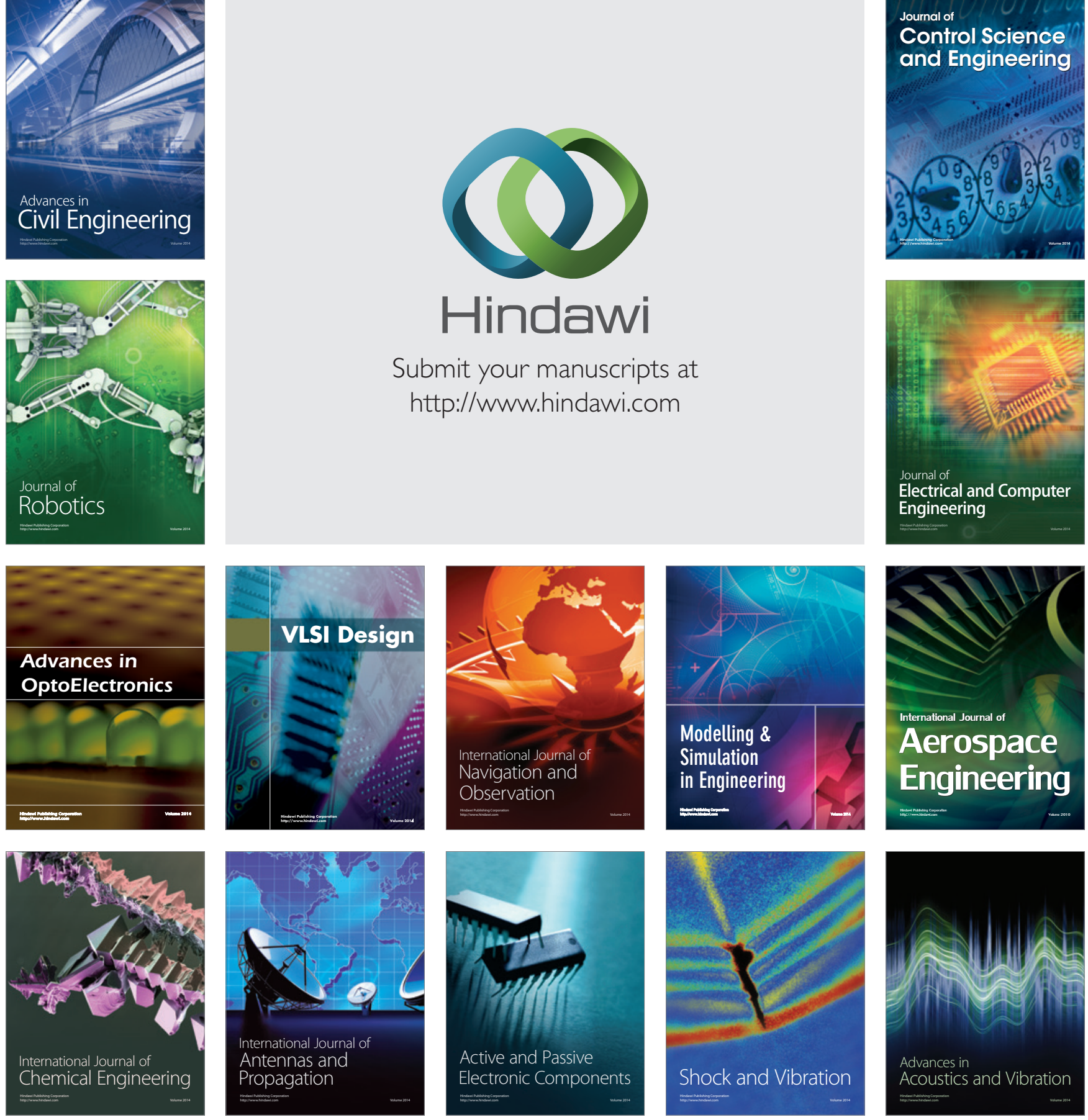